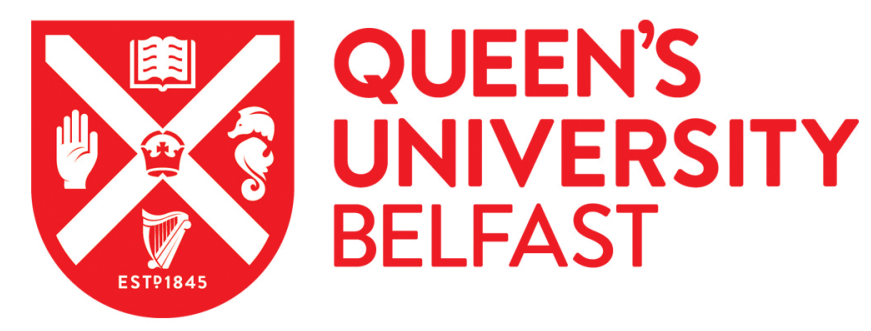

\title{
Detector for imaging and dosimetry of laser-driven epithermal neutrons by alpha conversion
}

Mirfayzi, S. R., Alejo, A., Ahmed, H., Wilson, L. A., Ansell, S., Armstrong, C., Butler, N. M. H., Clarke, R. J.,
Higginson, A., Notley, M., Raspino, D., Rusby, D. R., Borghesi, M., Rhodes, N. J., McKenna, P., Neely, D., Brenner, C. M., \& Kar, S. (2016). Detector for imaging and dosimetry of laser-driven epithermal neutrons by alpha conversion. Journal of Instrumentation, 11(10), C10008. https://doi.org/10.1088/1748-0221/11/10/C10008

Published in:

Journal of Instrumentation

Document Version:

Peer reviewed version

Queen's University Belfast - Research Portal:

Link to publication record in Queen's University Belfast Research Portal

\section{Publisher rights}

(c) 2016 IOP Publishing Ltd and Sissa Medialab srl.

This is an author-created, un-copyedited version of an article accepted for publication/published in Journal of Instrumentation. IOP

Publishing Ltd is not responsible for any errors or omissions in this version of the manuscript or any version derived from it. The Version of Record is available online at http://dx.doi.org/10.1088/1748-0221/11/10/C10008

\section{General rights}

Copyright for the publications made accessible via the Queen's University Belfast Research Portal is retained by the author(s) and / or other copyright owners and it is a condition of accessing these publications that users recognise and abide by the legal requirements associated with these rights.

Take down policy

The Research Portal is Queen's institutional repository that provides access to Queen's research output. Every effort has been made to ensure that content in the Research Portal does not infringe any person's rights, or applicable UK laws. If you discover content in the Research Portal that you believe breaches copyright or violates any law, please contact openaccess@qub.ac.uk. 


\title{
Detector for Imaging and Dosimetry of Laser-driven Epithermal Neutrons by Alpha Conversion
}

\author{
S. R. Mirfayzi, ${ }^{a}$ A. Alejo, ${ }^{a}$ H. Ahmed, ${ }^{a}$ L. A. Wilson, ${ }^{b}$ C. Armstrong, ${ }^{b, c}$ N.M.H. Butler, ${ }^{c}$ R. \\ J. Clarke, ${ }^{b}$ A. Higginson, ${ }^{c}$ M. Notley, ${ }^{b}$ D. Raspino, ${ }^{d}$ D. R. Rusby, ${ }^{b}$ M. Borghesi, ${ }^{a}$ N. \\ J. Rhodes, ${ }^{d}$ P. McKenna, ${ }^{c}$ D. Neely, ${ }^{b}$ C. M. Brenner, ${ }^{b}$ S. Kar ${ }^{a, 1}$ \\ ${ }^{a}$ Centre for Plasma Physics, School of Mathematics and Physics, Queen's University Belfast, BT71NN, UK \\ ${ }^{b}$ Central Laser Facility, Rutherford Appleton Laboratory, Didcot, Oxfordshire, OX11 OQX, UK \\ ${ }^{c}$ Department of Physics, SUPA, University of Strathclyde, Glasgow, G4 ONG, UK \\ ${ }^{d} I S I S$ Facility, Rutherford Appleton Laboratory, Chilton, Didcot, OX11 OQX, UK \\ E-mail: s.kar@qub.ac.uk
}

\begin{abstract}
An epithermal neutron imager based on detecting alpha particles created by boron neutron capture mechanism is discussed. The diagnostic mainly consists of a mm thick Boron Nitride (BN) sheet (as an alpha converter) in contact with a non-borated cellulose nitride film (LR115 type-II) detector. While the BN absorbs the neutrons below $0.1 \mathrm{eV}$, the fast neutrons register insignificantly on the detector due to their low neutron capture and recoil cross-sections. The use of solid-state nuclear track detectors (SSNTD), unlike image plates, micro-channel plates and scintillators, provide safeguard from the x-rays, gamma-rays and electrons. The diagnostic was tested on a proof-of-principle basis, in front of a laser driven source of moderated neutrons, which suggests the potential of using this diagnostic (BN+SSNTD) for dosimetry and imaging applications.
\end{abstract}

KeYwORDs: Instrumentation for neutron sources, Neutron detectors, Neutron radiography

\footnotetext{
${ }^{1}$ Corresponding author.
} 


\section{Contents}

1 Introduction 1

2 Mechanism 2

3 Experimental Setup $\quad 4$

4 Towards Tuning the Energy Response of the Diagnostic 5

5 Imaging 6

$\begin{array}{lll}6 & \text { Conclusion } & 7\end{array}$

$\begin{array}{lll}7 & \text { Acknowledgment } & 7\end{array}$

\section{Introduction}

In recent years the development of laser-driven neutron sources have demonstrated potentials for various applications due to their brightness, directionality and compactness[1]. Energetic (MeV) ions generated from intense laser matter interaction can efficiently drive nuclear fusion reactions in a secondary target (so called catcher), leading to the production of ultra-short (sub-ns) neutron pulses that can be employed in a wide range of applications in science [2], industry [3], security [4] and healthcare [5].

Neutrons generated from the laser driven sources are typically in the $\mathrm{MeV}$ energy region (known as fast neutrons). Although the fast neutrons are highly penetrating, for many neutron imaging applications epithermal neutrons $(1 \mathrm{eV}-100 \mathrm{KeV})$ are preferred, due to their excellent discriminatory capability between low and high z materials.

Imaging of epithermal neutrons is performed through several methods. The most preferred method is to convert neutrons into electrons or photons. Using a photosensitive film in contact with a conversion screen (such as ${ }^{155} \mathrm{Gd},{ }^{157} \mathrm{Gd}$ and ${ }^{113} \mathrm{Cd}$ ), the incoming neutrons are captured by the converter and the film is sensitised by the electrons produced from the conversion screen upon neutron capture [6]. A similar method employes scintillation screens and micro-channel plates (MCPs). In this case captured neutrons in the scintillation plate produce optical light and electrons, which are detected by the MCP [7] coupled to a camera by means of fibre optics leading to very high contrast radiographs.

A different mechanism is based on conversion of neutrons to heavy charged particles, such as alpha particles. The ions generated through this technique can be detected directly by image plates (IP), radiochromic films (RCF) and Solid State Track detectors (SSNTDs). While the IPs and RCFs are sensitive to electrons and gamma-rays, SSNTDs are capable of registering the passage of charged particles with no sensitivity to gamma-rays. This is an important advantage of this technique while 

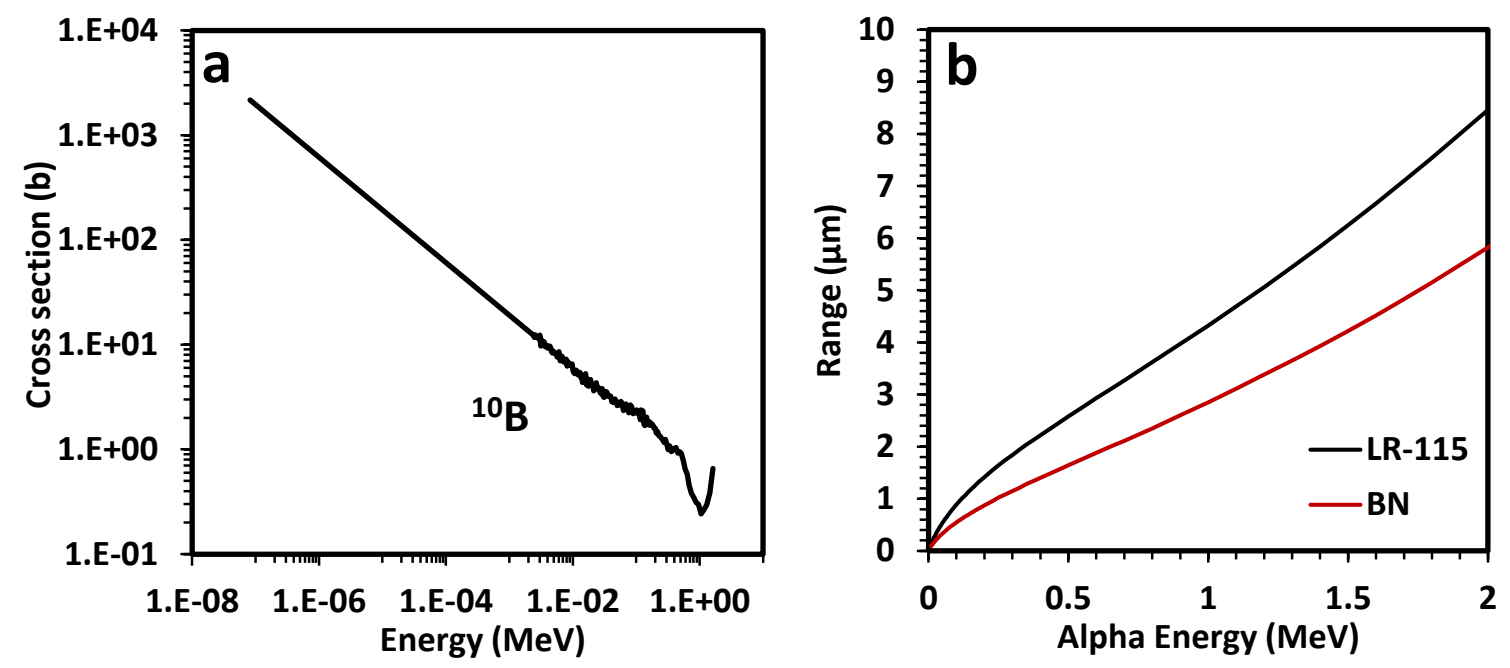

Figure 1. a) The cross section for alpha production in Boron-10 shows a linear trend. At lower neutron energies the alpha particle production is expected to be higher and it will be reduced as the neutron energy increases. b) SRIM simulation done for different alpha particle energies in Boron Nitride plate and LR-115 type-II film to measure the particle range. As expected the alphas created at the surface of $1 \mathrm{~mm}$ BN converter will be absorbed in very first layer and will not reach the detector.

deployed in a laser environment, where intense electron and gamma bursts produced by laser plasma interaction saturate almost every other type of detectors. When exposed, the impinging charged particles deposit energy along their trajectories inside the SSNTDs and the energy loss can lead to ionisation and breaking of molecular bonds. The decrease in molecular weight of the damaged region leads to its faster etching in an alkaline solution, compared to the surrounding undamaged material, creating conical pits for each incident ion [8].

In this paper we demonstrate imaging of epithermal neutron by alpha conversion via the ${ }^{10} B(n, \alpha){ }^{7} L i$ reaction and detection of alphas using SSNTD. In order to demonstrate the feasibility of the track-etched method for epithermal neutrons, alpha conversion has to be maximized at the neutron energy of interest. Therefore a thick Boron Nitride (BN) converter was used in order to suppress the response of the diagnostic for neutron energies below $0.1 \mathrm{eV}$. Not only does the thick BN layer help filtering sub-eV neutrons due to high capture cross-section at these energies, but the alpha particles thus created are also stopped inside the thick BN converter before reaching the SSNTD. This effect thus allows the possibility of tuning the spectral response of the diagnostic by varying the thickness of the $\mathrm{BN}$ converter layer. The diagnostic was fielded in a recent experimental campaign on a proof-of-principle basis, and the experimental data was reproduced by FLUKA simulation.

\section{Mechanism}

Neutron detection typically relies on elastic, inelastic scatterings or nuclear reactions. In the diagnostic discussed here, neutrons are detected via a two-step process -(1) neutron to alpha conversion via Boron neutron capture reaction and (2) detection of alphas by SSNTDs. The neutron 
capture reaction with ${ }^{10} \mathrm{~B}$, given by:

$$
n+{ }_{5}^{10} B \rightarrow\left\{\begin{array}{c}
{ }_{3}^{7} \mathrm{Li}^{*}(0.83 \mathrm{MeV})+{ }_{2}^{4} \mathrm{He}(1.47 \mathrm{MeV}) 93.7 \% \\
{ }_{3}^{7} \mathrm{Li}(0.01 \mathrm{MeV})+{ }_{2}^{4} \mathrm{He}(1.77 \mathrm{MeV}) 6.3 \%
\end{array}\right.
$$

which is one the most explored nuclear reaction and is the basis of one of the potential cancer treatment modality -the Boron Neutron Capture Therapy (BNCT) $[9,10]$. The cross-section of the $(\mathrm{n}$,alpha) reaction strongly depends on the incident neutron energy, as shown in Fig.1a, with the probability of alpha production becoming higher with lower neutron energies.

The alpha particles were produced by allowing the neutrons to impinge on a mm thick Pyrolitic Boron Nitride (chemical composition BN with $20 \%$ natural abundance of ${ }^{10} \mathrm{~B}$ nuclei). The (n,alpha) reaction produces alpha particles with energy $1.5 \mathrm{MeV}$, which has a stopping range of a few microns inside the BN converter, as obtained by SRIM monte-carlo simulation [11](shown in Fig.1b). Therefore the alpha particles produced close to the rear surface of the converter could be detected by using a SSNTD placed in contact with the rear side of the converter.

The small stopping range of the alpha particles, together with the large reaction cross-section for low ( $\sim 1 \mathrm{eV}$ and below) energy neutrons, provides a unique opportunity to discriminate low the energy neutrons by using a sufficiently thick converter material. Fig.2a shows FLUKA montecarlo [12] simulation of the alpha particle spectrum emerging from the rear surface of $1 \mathrm{~mm}$ thick BN foil for a range of incident neutron energies. The number of alpha particles created for different incident neutron energies can be obtained as shown in Fig.2b by integrating the alpha particle spectra shown in Fig.2a. As expected, the alpha particle production strongly depends on the incident neutron energy. For a thick $(1 \mathrm{~mm}) \mathrm{BN}$ converter, the alpha production shows a peak feature lying around $100 \mathrm{eV}$ region as a result of efficient neutron capture at the lower energies and reduced reaction cross-section for higher energies. This is in contrast to the case of using a ultra-thin $(1 \mu \mathrm{m})$ converter foil as shown in the Fig.2b, where a thin BN converter would provide a gradually declining production of alpha particles with increase in neutron energy, in agreement with the reaction cross-section shown in Fig.1a.

The alpha particles emerging from the converter have a continuous energy spectra up to $1.5 \mathrm{MeV}$, since the particles created deeper in the converter lose part of their energy inside the converter via electronic stopping. These alpha particles can be detected by different types of detectors, however, as discussed in the section 1, we used LR films due to their lack of sensitivity to the extraneous ionising radiations in the background, as well as their ease in handling. The polycarbonate and cellulose nitride plate (LR-115) SSNTDs were developed soon after the discovery of (n,alpha) reactions [13] in order to detect thermal and epithermal neutrons. Therefore the typically used LR film (LR115 - Type-I) contains a borated cellulose $\left(B_{10} H_{12} C_{2}\right)$ active layer for alpha particle creation and in-situ detection. However, the LR115 - Type-II was used in our case, which has a thicker (12 micron) layer of nonborated $\left(H_{12} C_{2}\right)$ active layer. The alpha particles in our case were produced by using the aforementioned thick BN layer in front of the LR film. The active layer of the LR films is highly sensitive to charged particles, and is designed especially for the detection of alpha particles. After exposure, the tracks created by the alpha particles can be revealed by etching in alkaline solution.

Having established the physics behind the alpha detection by the SSNTD one can also envisage the use of the technique for dosimetry applications. If the alpha particles are created with sufficiently 

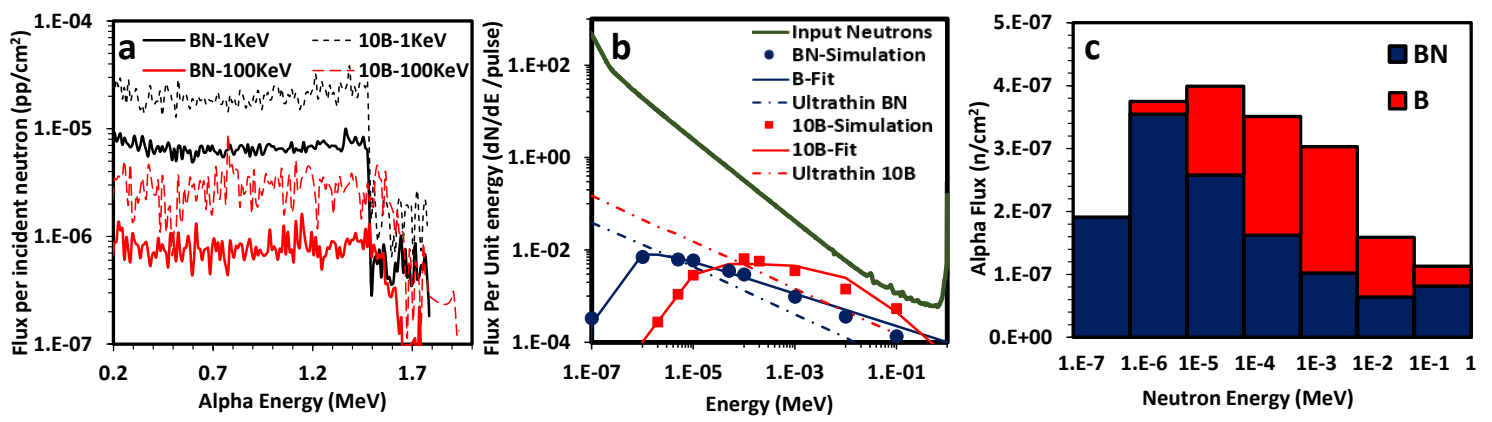

Figure 2. a) The FLUKA simulation performed for pure ${ }^{10} B$ and Pyrolitic Boron Nitride (BN) converters at different monoenergetic neutrons (i.e. $1 \mathrm{keV}$ and $100 \mathrm{KeV}$ ). As shown the ${ }^{10} B$ has the highest production yield due to the higher cross section. b) The blue circles are showing the simulated alpha particles exiting the converter rear surface at different input neutron energies. The alpha production for low neutron energy is significantly influenced by neutron penetration length. The blue dashed line shows that decreasing the BN thickness can lead to increase in alpha production at lower energies, however leads to lower efficiency of alpha production by epithermal neutrons. The green line is a typical neutron spectrum obtained from a polyethylene moderator, simulated using MCNPX. c) shows most of alpha particles are created by $1-10 \mathrm{KeV}$ for the $1 \mathrm{~mm}$ thick ${ }^{10} \mathrm{~B}$, compared with $\mathrm{BN}$ which had the highest yield for neutron energy in the range $1-10 \mathrm{eV}$. However using $1 \mathrm{~mm}$ thick ${ }^{10} \mathrm{~B}$ does not yield any neutron below $1 \mathrm{eV}$ due to its efficient neutron capture in the bulk.

high numbers, the use of SSNTD for dosimetry applications is well justified by counting the alpha tracks over a wide energy range to establish the dose received. The energy response of LR115 to different energy alpha particles is well measured (the detection efficiency is $20 \%-50 \%$ for $0.5 \mathrm{MeV}$ - $2 \mathrm{MeV}$ alphas $[14,15])$.

\section{Experimental Setup}

The diagnostic was fielded on a proof-of-principle basis in a recent experiment employing the VULCAN laser of the Rutherford Appleton Laboratory (RAL), STFC, UK [16]. The 750fs FWHM laser pulse with energy of 50J after compressor was focused on $10 \mu \mathrm{m}$ Au target by a f/3 off-axis parabola delivering peak intensity of $\sim 5 \times 10^{19} \mathrm{~W} \mathrm{~cm}^{-2}$ on to the target. The ion beams produced in interaction were allowed to impinge on a $\mathrm{LiCu}$ catcher in order to produce a beam of fast neutrons with flux of the order of $10^{9}$ above $1 \mathrm{MeV}$ [17]. The fast neutrons were moderated by using a thick (several $\mathrm{cm}$ ) block of plastic, following a thick (several $\mathrm{cm}$ ) block of lead along the fast neutron beam axis. A schematic of the setup is shown in Fig.3a.

A stack made of BN converter and LR film was used at the rear side of the plastic block. The design of the stack can be seen in the Fig. 3(a). In order to investigate the effectiveness of the diagnostic, a small $(2.5 \mathrm{~cm})$ square piece of $B N$ converter of $1 \mathrm{~mm}$ thick was placed on top of a $5 \mathrm{~cm}$ square piece of LR film. The stack was covered by a few mm thick lead layers on both sides of the $\mathrm{BN}+\mathrm{LR}$ stack, in order to protect the LR films from any stray particles. The energetic ions produced from the laser interaction are fully stopped by the catcher and the thick layer of lead in front of the plastic block. Although the other extraneous radiation, such as fast electrons and gamma rays, 

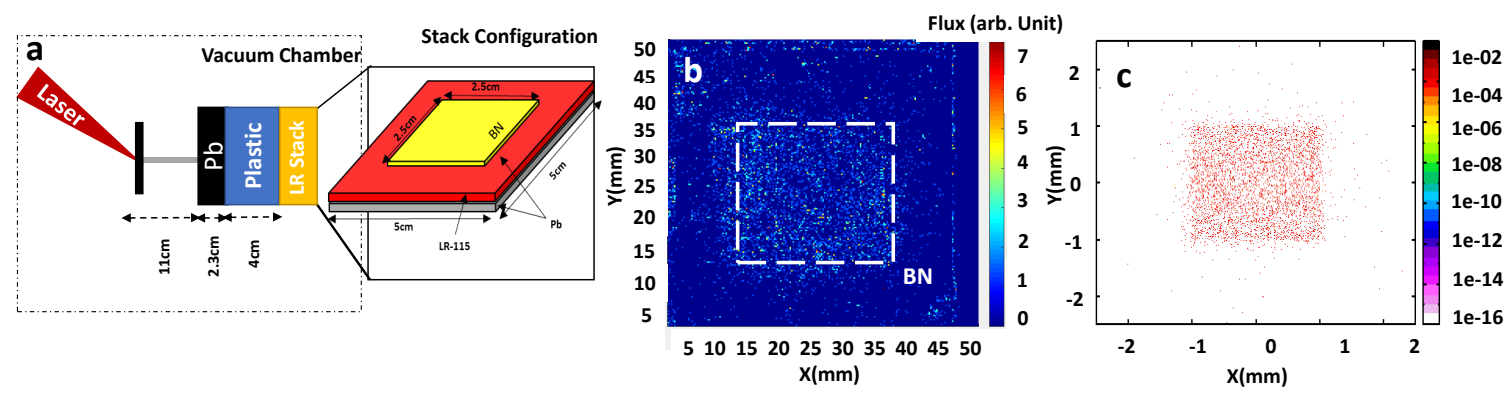

Figure 3. a) Experimental setup, showing a picture of the setup inside the interaction chamber. The fast neutrons were generated by nuclear reactions in the catcher target induced by the $\mathrm{MeV}$ ions accelerated from the laser irradiated pitcher target. Epithermal neutrons were produced by the plastic block was diagnosed by the BN+SSNTD stack, shown schematically in the insert.b) shows the experimental data recorded in LR-115 film. The dashed rectangle shows the position of the BN converter and the color scale represent the alpha particle flux on the detector. c) shows the results from FLUKA simulation showing similar alpha particle distribution on the detector film while using the same detector setup as in the experiment.

will also be strongly attenuated by the thick lead block, the LR films are extremely insensitive to photons and electrons. Therefore the only source of background signal in the LR film would be the fast neutrons. Therefore, the signal in the parts of the LR film not covered by the BN converter would provide a quantitative information about the background noise.

After exposure, the film was etched and scanned to count the number of pits created by the alpha particles produced via $(n, \alpha)$ reaction at the rear surface of the $\mathrm{BN}$ converter. These tracks were visible after chemical etching in $\mathrm{NaOH}$ solution at $60^{\circ} \mathrm{C}$ with $2.5 \mathrm{M}$ for 120 minutes. As shown in Fig.3b, the flux of alpha pits on the LR film was significantly higher in the region covered by the $\mathrm{BN}$ converter than elsewhere. The number of alpha pits on the LR film can be easily counted, from which the incident epithermal flux can be estimated by considering the detection efficiency of the LR film and the conversion efficiency of the the BN layer as discussed above. One can also use CR39 SSNTD instead of the LR film, which has a slightly higher alpha detection efficiency [18] compared to the LR film.

In order to substantiate the result obtained in the experiment, a FLUKA simulation was carried out exposing epithermal neutrons to a partially covered $5 \mathrm{~cm}$ by $5 \mathrm{~cm}$ detector film by an $2 \mathrm{~cm}$ by $2 \mathrm{~cm}$ BN layer. The simulation was set by incorporating the proper physics cards and using continuous cross section embedded in the simulation package. As can be seen in Fig.3c, the simulation closely reproduce the data obtained in the experiment shown in Fig.3b.

\section{Towards Tuning the Energy Response of the Diagnostic}

As discussed earlier, the response of the diagnostics also depends on the thickness of the converter. For an efficient detection of epithermal neutrons, the converter thickness must be thick enough so that the thermal neutrons can be captured inside the converter. Nevertheless, a thin layer of converter screen can be used for efficient detection of thermal neutrons due to the higher reaction cross-section for lower neutron energies. As can be seen in Fig.2b the energy response of a thin 

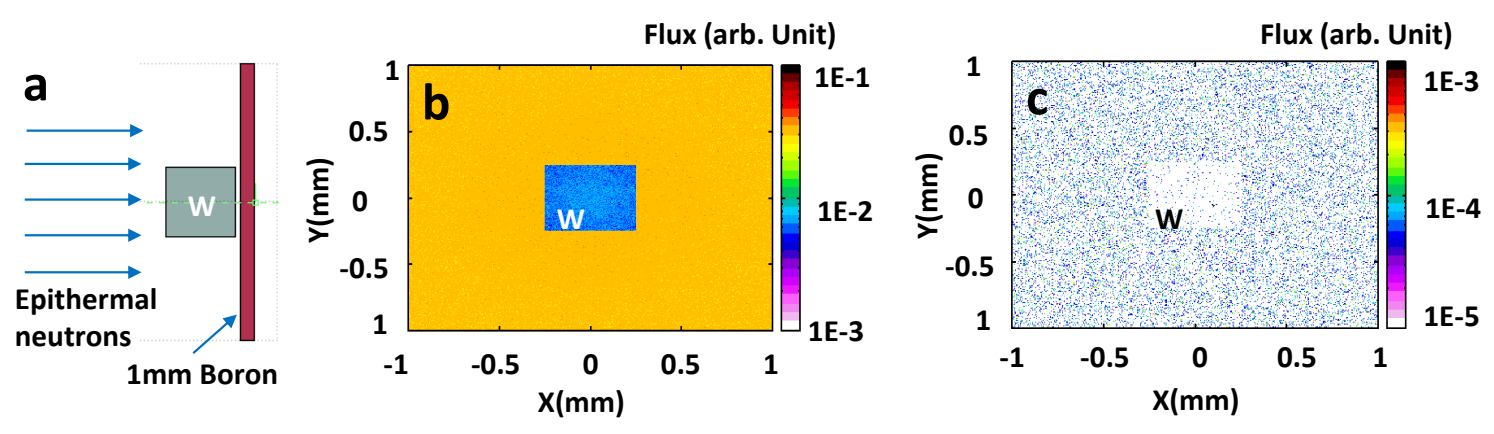

Figure 4. Neutron induced alpha radiography: a) shows how epithermal neutrons can be employed to probe objects using the difference in elastic cross section for different materials b-c) illustrates the FLUKA simulation done for Tungsten, shows very good contrast for the high neutron elastic cross section and atomic number.

converter is of similar trend as the boron neutron capture cross-section shown in Fig.1a. Using a thin layer of borated active layer is therefore the underlying principle of the LR-115 type I detector used for thermal neutron detection.

The other factor which can tune the energy response of the diagnostics is the material composition. For instance, a pure ${ }^{10} B$ converter layer, instead of the BN, would lead to higher alpha particle production, due to the significantly lower concentration of ${ }^{10} B$ nuclei in the $\mathrm{BN}$ (natural abundance of ${ }^{10} B$ is $20 \%$ ). For the same reason, a pure ${ }^{10} B$ converter screen would offer a significantly higher absorption for neutrons extended towards $\mathrm{eV}$ energy range. As can be seen in Fig.2b and Fig.2c, a $1 \mathrm{~mm}$ thick ${ }^{10} \mathrm{~B}$ converter can have the peak in the energy response curve shifted towards $\mathrm{keV}$ energies, as compared to the peak around $\mathrm{eV}$ energies for $1 \mathrm{~mm}$ thick $\mathrm{BN}$ layer.

\section{Imaging}

Epithermal neutrons are highly suitable for radiographic applications due to their excellent capability to penetrate through many materials and reveal their compositions due to the difference in their strong material dependent scattering cross sections. Since the neutron source obtained from a moderator is broadband, one would require a detector which has a good discrimination capability between thermal and epithermal neutrons. By considering the possibility of tuning the energy response of the diagnostic to the best region of the interest, one can employ the B+SSNTD as a suitable diagnostic for imaging applications. Fig.4 shows a model FLUKA simulation for a demonstration of the imaging capability using the diagnostic. A monoenergetic beam of $1 \mathrm{keV}$ neutron was used in this simulation for probing of a $0.5 \mathrm{~cm} \times 0.5 \mathrm{~cm}$ block of tungsten. The probe neutrons interacted with the atomic nuclei of tungsten and were elastically scattered, reducing the flux of neutrons in the shadow of the tungsten block compared to elsewhere (as shown in Fig.4b). The spatial variation in the neutron flux after probing the object will thus be commensurately converted to alpha particles, which can form the neutron radiograph on the SSNTD as shown in Fig.4c. Since the scattering cross-section depends both on the nucleus as well as incident neutron energy, contrast of the radiographs can be optimised by tuning the energy response of the diagnostic. 


\section{Conclusion}

A detector for epithermal neutron dosimetry and radiography was developed based on neutron to alpha conversion via boron neutron capture reaction. Using SSNTSs, with low sensitivity to gamma radiation, to detect the alpha particle gives a great advantage to any other type of detectors in extremely hostile environments, such as laser plasma interaction. Moreover, the ability to tune the energy response of the detector by varying the converter material is highly useful for precise dosimetry and high contrast neutron radiographic applications.

\section{Acknowledgment}

The authors acknowledge funding from EPSRC [EP/J002550/1-Career Acceleration Fellowship held by S. K., EP/L002221/1, EP/K022415/1 and EP/J500094/1. This work was also supported by STFC's Business and Innovation Directorate. Authors also acknowledge the support of ESG, mechanical and target fabrication staffs of the Central Laser Facility, STFC, UK.

\section{References}

[1] S. Kar et al. New J. Phys 18, 053002 (2016).

[2] K. W. D. Ledingham et al. Sci. 300, 1107 (2003).

[3] S. Nakai et al. J. of Phys.: Conf. Ser., 112, 042070 (2008)

[4] B.D. Sowerby, J.R. Tickner, Nucl.Inst. Met./ in Phys. Res. A 580, 799 (2007).

[5] A. Wittig, et. al., Crit. Rev. in Onc./Hema. 68, 66 (2008).

[6] H. D. Reis, Non-Destructive Testing And Evaluation For Manufacturing And Construction. CRC Press (1989).

[7] D. Ress, et. al., Rev. Sci. Ins. 66, 4943 (1995)

[8] R. L. Fleischer R. M. Walker., Nuclear Tracks in Solids: Principles and Applications, 626 (1975).

[9] R. Terlizzi et al., Appl. Rad. Isotopes, 67, S292 (2009)

[10] M.F. Hawthorne, Mol. Med. today, 4, 174 (1998)

[11] J.F. Ziegler, SRIM. Cadence Design Systems, 2008.

[12] A. Ferrari et al. FLUKA: A multi-particle transport code, INFN-TC-05-11 (2005).

[13] K. Becker ENEA. Symp. Rad. Dos. Meas., 317 (1967).

[14] D. Amrani and M. Belgaid Rad. Phys. Chem., 61.3639 (2001).

[15] K. Ashkok et. al., Def. Sci. Jour., 42.4, 227 (1992).

[16] C. Danson et al. Nucl. Fus. 44, S239 (2004).

[17] C. M. Brenner et. al. Plasm. Phys. Cont. fus. 58.1, 014039 (2015).

[18] M.A. Misdaq et al., / Nucl. Instr. and Meth. in Phys. Res. B, 171, 350 (2000). 